\title{
Motivación y rendimiento académico en matemáticas: un estudio longitudinal en las primeras etapas educativas
}

\section{Motivation and academic achievement in mathematics: a longitudinal study in the early stages of education}

\author{
Número de palabras (6397)
}

Resumen

Se analiza longitudinalmente la capacidad predictiva de un conjunto de variables motivacionales sobre el rendimiento matemático. Los análisis se realizan, en una muestra de 180 sujetos, con datos evaluados en dos momentos (Educación Infantil 5 años y $2^{\circ}$ curso de Primaria). También se estudian las diferencias entre dos grupos, con rendimiento matemático medio y bajo al finalizar el primer ciclo de Primaria, en las variables motivacionales evaluadas en el primer momento. Los resultados muestran un importante poder predictivo sobre el rendimiento matemático posterior de la competencia percibida. También la persistencia, la actitud y la dimensión atribucional de internalidad positiva aportan una contribución significativa adicional. Los resultados muestran igualmente que los grupos con rendimiento medio y bajo al finalizar $2^{\circ}$ de Primaria presentaban diferencias significativas a los 5 años, tanto en internalidad positiva como en persistencia. Se discuten las implicaciones teóricas y prácticas de los resultados.

Palabras clave: motivación, estilo atribucional, autocompetencia, rendimiento matemático.

\section{Abstract}

This longitudinal study analyzes the predictive value of a set of motivational variables on academic performance in mathematics area. Analyses were performed, in a sample of 180 subjects, with the data evaluated in two stages in formal educational settings (from kindergarten through 2nd grade of Primary School). Likewise, differences between groups, with average (AM) and low math performance (LM) at the end of the second year of Primary School, in motivational and attributional variables assessed at the first moment, are also studied. The results show a significant predictive power on later mathematics achievement for self-competence. Also persistence, attitude and positive attributional dimension of internality have an additional significant contribution. The results also show that average and low yield groups at the end of 2nd grade of Primary School, showed significant differences at 5 years both positive internality and persistence. These results are discussed in terms of their theoretical and practical implications.

Keywords: motivation, attributional style, self-competence, mathematic performance. 


\section{Introducción}

Diferentes estudios han establecido una serie de habilidades que parecen estar relacionadas con el rendimiento y las dificultades en el área de matemáticas. Estos factores, pueden tener un carácter específico para el desarrollo y la aplicación de estrategias en tareas matemáticas o enmarcarse dentro de componentes superiores más generales que tienen influencia sobre el aprendizaje (Passolunghi y Lanfranchi, 2012). Así, los factores específicos conciernen a habilidades matemáticas básicas, tales como las competencias de conteo, las habilidades lógicas o la numerosidad (Desoete y Grégoire, 2006). Entre los componentes generales podemos encontrar factores de tipo cognitivo, tales como el CI o las funciones ejecutivas de inhibición, memoria de trabajo o flexibilidad, que han demostrado ser predictores del rendimiento matemático a lo largo del curso evolutivo (Bull y Lee, 2014) tanto si son evaluadas con medidas neuropsicológicas como mediante estimaciones ecológicas (Autores, 2015).

Los enfoques actuales sobre el aprendizaje ponen de manifiesto igualmente la necesidad de integrar factores motivacionales en la explicación del rendimiento matemático (Op’tEynde, De Corte y Verschaffel, 2006; Sarabia e Iriarte, 2011). Desde esta perspectiva, una adecuada motivación es necesaria para la regulación de las estrategias cognitivas y metacognitivas que requiere un aprendizaje matemático significativo (Ugartetxea, 2002).

La motivación hacia el aprendizaje se define como un proceso que nos impulsa a aprender, una disposición que instiga y mantiene el interés hacia los elementos de aprendizaje que se presentan (Pintrich y Schunk, 2006; Wigfield y Eccles, 2002). La relación entre la motivación hacia el aprendizaje y el rendimiento matemático ha sido demostrada con muestras de niños de Educación Primaria (McKenzie, Gow, y Schweitzer, 2004; Pinxten, Marsh, De Fraine, Van Der Noortgate, y Van Damme, 2014) y Secundaria (Moenikia y Zahed-Babelan, 2010; Suárez-Álvarez, Fernández, y Muñiz, 2014). En muestras de niños menores de 7 años, la investigación es mucho más reducida y algunos autores han operacionalizado este tipo de motivación en base a un conjunto de conductas de aprendizaje de carácter observable ("learning behaviors"), relacionadas con el esfuerzo y el grado de compromiso dirigidos a una meta que los alumnos muestran ante las actividades del aula (veáse McDermott, Green, Francis, y Stott, 2000). Estudios en esta línea muestran que la contribución de la motivación inicial hacia el aprendizaje no parece recaer únicamente sobre el rendimiento matemático inmediato (Daniels, 2014), sino también en la ejecución matemática en cursos posteriores (Ladd, Buhs, y Seid, 2000; Mokrova, O’Brien, Calkins, Leerkes, y Mcrovitch, 2013; Reimann et al., 2013).

Destaca especialmente la investigación del grupo de McDermott y sus colaboradores con niños de familias desfavorecidas. Sus estudios muestran que aquellos comportamientos que reflejan aspectos como autocompetencia percibida, persistencia ante los errores o actitud hacia los elementos del aprendizaje en Educación Infantil poseen un especial impacto en la predicción del rendimiento matemático posterior (Fantuzzo, Perry, y McDermott, 2004; McDermott, Mordell, y Stoltzfus, 2001), aumentando sustancialmente el poder predictivo de la habilidad cognitiva general (Yen, Konold, y McDermott, 2004). Es más, parece que dichas conductas, especialmente las relacionadas con la capacidad inicial para anticipar el éxito en base a la competencia percibida, constituyen un factor de protección hacia posibles dificultades de aprendizaje matemáticas posteriores (McDermott, Goldberg, Watkins, Stanley, y Glutting, 2006; McDermott et al., 2011). En este sentido, los resultados de un reciente trabajo evidencian 


\section{MOTIVACIÓN Y RENDIMIENTO ACADÉMICO EN MATEMÁTICAS: UN3 ESTUDIO LONGITUDINAL EN LAS PRIMERAS ETAPAS EDUCATIVAS}

la sensibilidad de las habilidades iniciales de competencia percibida y persistencia ante las dificultades para la diferenciación entre las trayectorias de niños con y sin problemas de rendimiento matemático desde preescolar hasta segundo curso de Educación Primaria (McDermott, Rikoon, y Fantuzzo, 2014).

Por otra parte, el estilo atribucional es descrito como la percepción que los sujetos tienen acerca de las causas de los acontecimientos que les suceden a sí mismos y a otros individuos. Weiner (1986) postula que el comportamiento motivado está influido por las expectativas de logro de una meta y el valor que se le otorga. Estos, a su vez, vienen determinados por las atribuciones expresadas mediante las creencias personales sobre cuáles son las causas originarias tanto de los éxitos como de los fracasos. Las personas podemos atribuir los acontecimientos que nos suceden a causas internas o externas, estables o inestables y puntuales o globales. Estas dimensiones poseen implicaciones motivacionales sobre las conductas futuras y el aprendizaje. La relación entre las atribuciones y el proceso general de aprendizaje ha sido descrita en términos de implicación del feedback atribucional en la autoregulación del aprendizaje y los resultados del mismo (Pintrich y Schunk, 2006).

Los estudios realizados con muestras de Educación Primaria y mayores muestran que la atribución del éxito a causas internas y estables parece determinar un mejor rendimiento escolar (Miñano y Castejón, 2011). Las evidencias en cuanto a las situaciones de fracaso son más claras. Según Miñano y Castejón (2011) son las atribuciones a causas inestables y controlables las que han mostrado ser más adaptativas $\mathrm{y}$, por lo tanto, atribuir el fracaso a causas internas, estables y/o no controlables produce efectos negativos tanto sobre las futuras expectativas de éxito (Closas, Sanz de Acedo y Ugarte, 2011; González, 2005), como sobre el rendimiento final (Lozano, Pezzutti, Blanco, y Canosa, 2000).

Información adicional ha sido aportada por trabajos que se han centrado en niños con dificultades de aprendizaje de las matemáticas. La mayoría evidencia que los sujetos con un diagnóstico clínico de dificultades específicas presentan una mayor probabilidad de manifestar déficits en dichos factores. Así, la revisión de Miranda, García, Marco, y Rosel (2006) concluye que los sujetos con dificultades específicas en el aprendizaje de las matemáticas, en comparación con los estudiantes con un rendimiento matemático adecuado, suelen mostrar un estilo motivacional extrínseco y atribuyen en menor medida sus éxitos y fracasos al interés y al esfuerzo personal. En esta misma línea se encuentran los resultados de un reciente trabajo realizado por Pasta, Mendola, Logonbardi, Prino y Gastaldi (2013), en el que los alumnos con dificultades (específicas o bajo rendimiento en matemáticas y/o lectura) atribuyen en menor medida los éxitos y los fracasos a causas internas. No obstante, algunos estudios muestran que dicho estilo atribucional desadaptativo parece no estar presente en todos los alumnos con dificultades de aprendizaje (González-Pienda et al., 2000; Núñez et al., 2005).

A modo de conclusión, parece que distintos trabajos apuntan hacia la importancia de las variables motivacionales sobre el rendimiento matemático y sus dificultades, aunque se han centrado en niños mayores. Los estudios que han tratado de valorar el estilo atribucional utilizan muestras de Educación Primaria y Secundaria (más de 8 años). En relación a la motivación hacia el aprendizaje, la gran mayoría de los estudios con niños pequeños se han realizado dentro del programa "Head Start Children" (Department of Health and Human Services, USA), con familias de nivel socioeconómico bajo. Es necesario continuar profundizando en estos aspectos motivacionales con una perspectiva 
longitudinal que abarque el desarrollo de la competencia matemática desde las primeras etapas educativas.

En virtud de lo anteriormente expuesto, el presente trabajo pretende examinar la relación de la motivación hacia el aprendizaje y el estilo atribucional con el rendimiento matemático y sus dificultades en las primeras etapas escolares. Concretamente, se proponen dos objetivos diferenciados: a) analizar el poder predictivo de la motivación hacia el aprendizaje y el estilo atribucional evaluados en Educación Infantil sobre el rendimiento matemático en $2^{\circ}$ de Educación Primaria; y b) Examinar las diferencias en dichos factores motivacionales entre grupos con $\mathrm{y}$ sin problemas de rendimiento matemático en $2^{\circ}$ de Educación Primaria.

\section{Método}

\section{Participantes}

Con la finalidad de abarcar un amplio rango de centros, se seleccionaron 6 alumnos por aula al azar mediante la técnica del muestreo aleatorio simple. La muestra inicial, en el tiempo 1 (T1), estuvo conformada por 209 preescolares (52.2\% varones; 47.8\% niñas) de 5 y 6 años (Media $=70.17$ meses; DT $=3.51$ meses). Los sujetos presentaban una media de CI equivalente de 98.63 meses (DT $=12.23$ ), que se obtuvo a través las subpruebas vocabulario y cuadrados de la escala WPPSI (Wechsler, 1996) siguiendo las directrices de Spreen y Strauss (1991). Se excluyeron de la muestra aquellos sujetos que presentaban un CI equivalente inferior a 70, así como los niños con informes escolares de deficiencias sensoriales graves, anomalías neurobiológicas, trastornos psicológicos o deprivación socio-cultural. El $88 \%$ de los participantes tenía nacionalidad española, siendo el resto provenientes de otros países. El $63.6 \%$ de niños asistían a centros públicos y el $36.4 \%$ a colegios concertados.

Dos años después (tiempo 2/T2), se evaluó a la muestra definitiva del estudio, conformada por 180 sujetos ( $86.6 \%$ de la muestra inicial). No se encontraron diferencias en las variables fundamentales para el estudio entre los sujetos que formaron parte de la muestra en el T2 y aquellos que se perdieron, evitando así que los datos resultantes de la presente investigación estuvieran sesgados por la pérdida longitudinal. Los participantes presentaban en esta fase edades comprendidas entre los 7 y los 8 años (Media $=94.16$ meses; DT $=3.78$ meses). El $51.1 \%$ de los sujetos de la muestra final eran varones, siendo el $48.9 \%$ niñas. El $87.8 \%$ poseía nacionalidad española. En cuanto a la titularidad de los centros, el $65 \%$ de los sujetos asistía a centros públicos, frente al 35\% que pertenecía a colegios concertados. El $19.1 \%$ de los participantes asistía a sesiones con especialistas en los respectivos centros escolares en el T2: Apoyo Educativo (7.7\%), Educación Compensatoria (1.9\%), Pedagogía Terapéutica (3.3\%), Audición y Lenguaje (3.8\%) y tratamiento combinado $(2.4 \%)$.

Para el segundo objetivo, la muestra se dividió en dos grupos en función del Índice de Competencia Matemática (ICM) obtenido en la prueba TEMA-3 en $2^{\circ}$ curso de Educación Primaria. El grupo con rendimiento medio (RM) estaba compuesto por 66 sujetos, con un Índice de Competencia Matemática (ICM) de 90-110 (descriptor medio) correspondiente a un rango percentil de 25-75. Se categorizaron en el grupo de rendimiento bajo (RB) 33 sujetos, 18 de los cuales obtuvieron un ICM de rango 70-79 (descriptor pobre) equivalente al percentil <1-6; y 15 con un ICM $<70$ (descriptor muy pobre) correspondiente al percentil $<1$. 
Se encontraron diferencias estadísticamente significativas entre los grupos de rendimiento en las variables edad en el T1 $[t(82.33)=-2.07 ; p=.042]$ y CI Equivalente $[t(97)=2.12 ; p=.036]$ que fueron tenidas en cuenta en posteriores análisis.

\section{Instrumentos}

\section{Tiempo 1: Educación Infantil 5 años}

Los profesores cumplimentaron Escala de Conductas de Aprendizaje en Preescolar (Preeschool Learning Behaviors Scale, PLBS; McDermott et al., 2000). Comprende 29 ítems que se agrupan en tres subescalas: competencia-motivación, que abarca conductas relacionadas con la anticipación del éxito (e.g. "Parece que se refugia en una actitud de impotencia"); atención-persistencia, cuyos ítems se centran en la capacidad de persistir en una tarea hasta completarla (e.g. "Se implica en las tareas en la medida que se espera que lo hiciera para su edad"); y actitud hacia el aprendizaje, que indica la voluntad de participar en actividades de aprendizaje, mostrando una actitud positiva hacia los elementos que lo componen (e.g. "Muestra poco interés en agradar al profesor"). Se constató el nivel de fiabilidad de la escala para la presente muestra (Alfa de Cronbach = .86). Se utilizó la puntuación directa en cada una de las subescalas para los análisis.

Igualmente, se administró individualmente la Entrevista del Estilo Atribucional para Niños (Children's Attributional Style Interview, CASI; Conley, Haines., Hilt, y Mestalky, 2001) aplicable a niños a partir de 5 años. En esta tarea, se presentan al sujeto una serie de ilustraciones (16 historias) de eventos relacionados con el rendimiento. El niño debe generar sus propias atribuciones y valorarlas en términos de internalidad ("depende de mí" vs. "depende de otros") globalidad ("ocurre en todas partes" vs. "ocurre sólo en un escenario concreto") y estabilidad ("sucede muchas veces" vs. "sucede sólo esta vez"). La mitad de las historias son positivas y, el resto, negativas. La prueba posee indicadores de fiabilidad y validez (Conley et al., 2001). Se tomaron como referencia el sumatorio de las respuestas en cada uno de los índices del cuestionario.

\section{Tiempo 2: $2^{\circ}$ Curso de Educación Primaria}

Para evaluar el rendimiento matemático se aplicó el Test de Competencia Matemática Básica (TEMA-3; Ginsburg y Baroody, 2003). Se trata de una prueba estandarizada dirigida a sujetos entre 3 años y 8 años y 11 meses. Se compone de 72 ítems que valoran diferentes aspectos de la competencia matemática infantil. Contempla tanto aspectos informales (aquellos que no requieren el uso de símbolos matemáticos escritos), que son evaluados mediante 41 ítems, como aspectos formales (actividades que implican el uso de símbolos matemáticos), que se engloban en 31 ítems. Todos ellos se agrupan en 8 dimensiones: cuatro subescalas relativas a las habilidades informales de numeración, comparación, cálculo y conceptos; cuatro subescalas de habilidades formales sobre convencionalismos, hechos numéricos, cálculo y conceptos. La prueba cuenta con elevados índices de fiabilidad y validez (Ginsburg y Baroody, 2003). Para el presente estudio, se utilizaron las puntuaciones directas en cada una de las subescalas y la puntuación total. Para el segundo objetivo, los sujetos se clasificaron en función del ICM.

\section{Procedimiento}


Tras obtener los permisos pertinentes, la evaluación fue llevada a cabo por profesionales familiarizados con la aplicación y corrección de los tests. La administración fue individual, en el tiempo medio de aplicación que se establece en el manual y respetando ritmos individuales. Los espacios físicos reunían las condiciones de iluminación, ventilación e insonorización adecuadas para la evaluación.

En el tiempo 1, se administró el cuestionario CASI (Conley et al., 2001) a modo de entrevista a los alumnos de Educación Infantil 5 años en horario lectivo sin interferir en las actividades significativas del currículum y a lo largo del tercer trimestre del curso escolar. Asimismo, los maestros cumplimentaron la escala de estimación PLBS (McDermott et al., 2000). Los cuestionarios fueron entregados en sobres cerrados a los profesores y se retornaron a los experimentadores.

Dos cursos escolares después, en el tiempo 2, se volvió a los centros escolares donde se administró la prueba estandarizada TEMA-3 (Gingsburg y Baroody, 2003) a los mismos sujetos, siguiendo el mismo procedimiento descrito anteriormente.

\section{Análisis estadísticos}

Se utilizó paquete estadístico Statistical Package for the Social Science (SPSS), versión 22.00 (SPSS Inc., Chicago, IL USA). Para el primer objetivo, se realizaron análisis de regresión lineal múltiple por el método de pasos sucesivos para comprobar qué conductas motivacionales y dimensiones de estilo atribucional a los 5 años predecían el rendimiento matemático posterior en $2^{\circ}$ curso de Educación Primiara.

Para el segundo objetivo, se realizaron análisis multivariados de la covarianza (MANCOVA's), introduciendo como covariables el CI y la edad en el tiempo 1, para establecer comparaciones entre los grupos con RM y RB de $2^{\circ}$ curso de Educación Primaria con las variables motivacionales y atribucionales evaluadas en Educación Infantil. El nivel de significación se fijó en .05 y se calculó $\eta^{2} p$ para comprobar la fuerza de asociación.

\section{Resultados}

\section{Poder predictivo de la motivación hacia el aprendizaje y el estilo atribucional de Infantil en el rendimiento matemático de $2^{\circ}$ de Primaria}

En la Tabla 1 se muestran los resultados sobre qué conductas motivacionales evaluadas en Educación Infantil predecían el rendimiento matemático en $2^{\circ}$ curso de Educación Primaria.

La puntuación total de la prueba TEMA-3 fue predicha en un $28.2 \%$ por las variables competencia-motivación $\left(\Delta R^{2}=.213, p<.001\right)$, actitud $\left(\Delta R^{2}=.049, p=.001\right)$ $\mathrm{y}$ atención-persistencia $\left(\Delta R^{2}=.020, p=.033\right)$.

Respecto a las habilidades informales, la tarea numeración es predicha por las variables competencia-motivación $\left(\Delta R^{2}=.153, p<.001\right)$, la atención-persistencia $\left(\Delta R^{2}\right.$ $=.040, p=.003) \mathrm{y}$ la actitud hacia el aprendizaje $\left(\Delta R^{2}=.019, p=.044\right)$, con un peso de un $21.2 \%$ de la varianza. Únicamente la variable competencia-motivación predice la subprueba comparación en un $15.1 \%$ de la varianza total $\left(\Delta R^{2}=.151, p<.001\right)$. La competencia-motivación $\left(\Delta R^{2}=.123, p<.001\right)$, la atención-persistencia $\left(\Delta R^{2}=.025, p\right.$ $=.023)$ y la actitud $\left(\Delta R^{2}=.022, p=035\right)$ explican un $16.9 \%$ de la varianza en cálculo. 


\section{MOTIVACIÓN Y RENDIMIENTO ACADÉMICO EN MATEMÁTICAS: UN7 ESTUDIO LONGITUDINAL EN LAS PRIMERAS ETAPAS EDUCATIVAS}

Únicamente actúa como predictor de la subprueba conceptos informal la variable atención-persistencia $\left(\Delta R^{2}=.128, p<.001\right)$ que explica el $12.8 \%$ de la varianza.

Respecto a las habilidades formales, la subprueba convencionalismos es predicha con un $12.6 \%$ de la varianza por la variable competencia-motivación $\left(\Delta R^{2}=.126, p<\right.$ $.001)$. La variable competencia-motivación explica también el $18.5 \%$ de la varianza en la subprueba hechos numéricos $\left(\Delta R^{2}=.185, p<.001\right)$. Los resultados obtenidos en cálculo los predicen atención-persistencia $\left(\Delta R^{2}=.213, p<.001\right)$ y actitud $\left(\Delta R^{2}=.050, p=.001\right)$ que explican el $26.3 \%$ de la varianza total. Finalmente, se observa que el $17.7 \%$ de la varianza de la variable conceptos formal lo explican la competencia-motivación $\left(\Delta R^{2}=\right.$ $.133, p<.001)$, la atención-persistencia $\left(\Delta R^{2}=.025, p=.022\right)$ y la actitud $\left(\Delta R^{2}=.019\right.$, $p=.046)$.

Tabla 1

Análisis de regresión de las subescalas del cuestionario de conductas de motivación hacia el aprendizaje (PLBS) sobre las medidas de rendimiento matemático (TEMA-3)

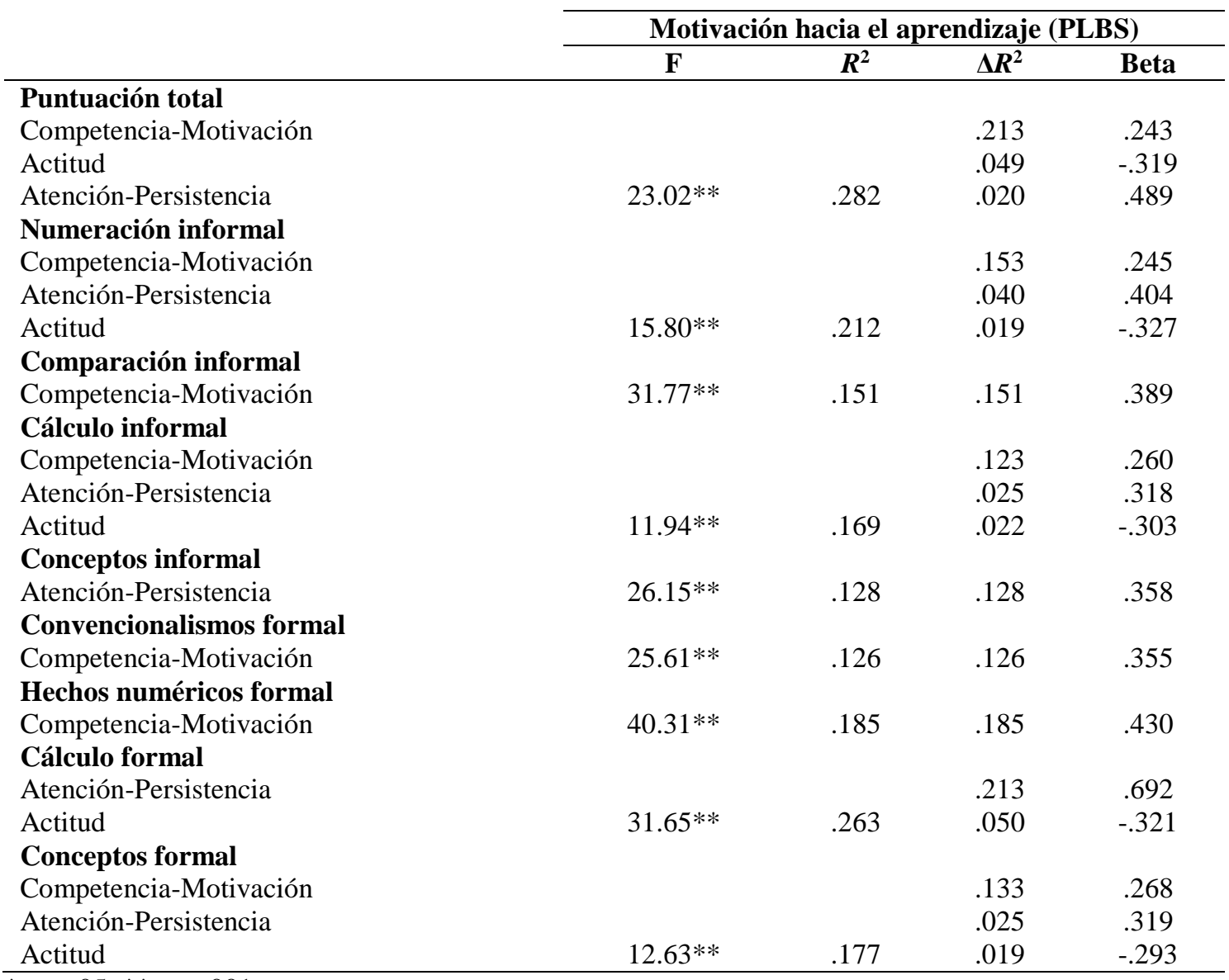

$* p<.05 ; * * p<.001$

En la Tabla 2 se presentan los resultados del análisis de regresión lineal múltiple por pasos sucesivos para las variables de estilo atribucional del último curso de Educación Infantil con respecto a las habilidades matemáticas evaluadas dos años más tarde.

La variable internalidad ante eventos positivos resultó el único predictor estadísticamente significativo para las puntuaciones obtenidas en las diferentes subpruebas de rendimiento matemático, con varianzas que oscilan entre el $2.2 \%$ y el 
4.1\%. Los resultados obtenidos en los análisis de regresión lineal son: puntuación total $\left(\Delta R^{2}=.041, p=.006\right)$, numeración informal $\left(\Delta R^{2}=.035, p=.012\right)$, comparación informal $\left(\Delta R^{2}=.030, p=.019\right)$, cálculo informal $\left(\Delta R^{2}=.033, p=.015\right)$, conceptos informal $\left(\Delta R^{2}\right.$ $=.034, p=.013)$, hechos numéricos formales $\left(\Delta R^{2}=.034, p=.014\right)$, cálculo formal $\left(\Delta R^{2}\right.$

$=.031, p=.018) \mathrm{y}$ conceptos formales $\left(\Delta R^{2}=.022, p=.046\right)$.

Tabla 2

Análisis de regresión de las dimensiones de estilo atribucional (CASI) sobre las medidas de rendimiento matemático (TEMA-3).

\begin{tabular}{lcccc} 
& \multicolumn{4}{c}{ Estilo atribucional (CASI) } \\
\hline & $\mathbf{F}$ & $\boldsymbol{R}^{\mathbf{2}}$ & $\boldsymbol{\Delta R}^{\mathbf{2}}$ & Beta \\
\hline $\begin{array}{l}\text { Puntuación total } \\
\text { Internalidad positiva }\end{array}$ & $7.61^{*}$ & .041 & .041 & .203 \\
$\begin{array}{l}\text { Numeración Informal } \\
\text { Internalidad positiva }\end{array}$ & $6.45^{*}$ & .035 & .035 & .187 \\
$\begin{array}{l}\text { Comparación Informal } \\
\text { Internalidad positiva }\end{array}$ & $5.56^{*}$ & .030 & .030 & .174 \\
$\begin{array}{l}\text { Cálculo informal } \\
\text { Internalidad positiva }\end{array}$ & $6.07^{*}$ & .033 & .033 & .182 \\
$\begin{array}{l}\text { Conceptos Informal } \\
\text { Internalidad positiva }\end{array}$ & $6.36^{*}$ & .034 & .034 & .186 \\
$\begin{array}{l}\text { Convencionalismos Formal } \\
\text { Hechos numéricos Formal }\end{array}$ & $n . s$. & - & - & - \\
$\begin{array}{l}\text { Internalidad positiva } \\
\text { Cálculo Formal }\end{array}$ & $6.22^{*}$ & .034 & .034 & .184 \\
$\begin{array}{l}\text { Internalidad positiva } \\
\text { Conceptos Formal } \\
\text { Internalidad positiva }\end{array}$ & $5.72^{*}$ & .031 & .031 & .176 \\
\hline
\end{tabular}

$* p<.05 ; * * p<.001$

Diferencias entre grupos con y sin problemas de rendimiento matemático en $2^{\circ}$ de Educación Primaria en factores motivacionales

La Tabla 3 muestra las diferencias en las variables motivacionales y atribucionales evaluadas en Educación Infantil para los grupos con RB y RM en $2^{\circ}$ de Educación Primaria.

El efecto principal de grupo resultó estadísticamante significativo para las variables de motivación hacia el aprendizaje [Wilks' Lambda $(\Lambda)=.89, \mathrm{~F}(3,93)=3.61, p$ $\left.=.016, \eta^{2} \mathrm{p}=.104\right]$. Los ANCOVA's de confirmación mostraron la existencia de diferencias estadísticamente significativas entre los grupos con RM y RB en la variable atención-persistencia: $\mathrm{F}(1,95)=9.49, p=.003, \eta^{2} \mathrm{p}=.091$. No se encontraron diferencias en el resto de variables motivacionales analizadas.

Respecto a las variables atribucionales, el efecto principal del grupo resultó estadísticamente significativo [Wilks' Lambda $(\Lambda)=.87, \mathrm{~F}(6,90)=2.31, p=.041, \eta^{2} \mathrm{p}$ $=.133]$. Los ANCOVA's de confirmación mostraron diferencias significativas entre el grupo con RM y el grupo con RB en la variable internalidad positiva $(\mathrm{F}(1,95)=4.71, p$ $\left.=.003, \eta^{2} \mathrm{p}=.047\right)$. No se encontraron diferencias en el resto de dimensiones del estilo atribucional.

Tabla 3 


\section{MOTIVACIÓN Y RENDIMIENTO ACADÉMICO EN MATEMÁTICAS: UN9 \\ ESTUDIO LONGITUDINAL EN LAS PRIMERAS ETAPAS EDUCATIVAS}

Análisis de comparación de medias entre los grupos Rendimiento Bajo y Rendimiento medio en motivación hacia el aprendizaje y estilo atribucional.

\begin{tabular}{lccccc}
\hline & $\mathbf{R B}$ & $\mathbf{R M}$ & & & \\
& $\mathbf{N}=\mathbf{3 3}$ & $\mathbf{N = 6 6}$ & & & \\
\hline PLBS & & $\mathbf{M}(\mathbf{D T})$ & $\mathbf{F}(\mathbf{1 , 9 5})$ & $\boldsymbol{p}$ & $\mathbf{\eta 2 p}$ \\
Competencia- & $16.18(3.94)$ & $18.09(4.91)$ & 3.38 & .069 & .034 \\
Motivación & $10.85(3.42)$ & $13.74(4.38)$ & 9.49 & .003 & .091 \\
Atención-Persistencia & $11.55(2.21)$ & $12.33(2.20)$ & 2.46 & .120 & .025 \\
Actitud & & & & & \\
CASI & & & & \\
Internalidad positiva & $4.67(1.14)$ & $5.27(1.24)$ & 4.71 & .032 & .047 \\
Estabilidad positiva & $5.64(2.16)$ & $6.27(1.78)$ & 2.49 & .117 & .026 \\
Globalidad positiva & $6.09(1.73)$ & $6.55(1.31)$ & 1.31 & .254 & .014 \\
Internalidad negativa & $3.64(1.59)$ & $3.42(1.60)$ & .289 & .592 & .003 \\
Estabilidad negativa & $3.61(2.47)$ & $3.52(2.56)$ & .072 & .784 & .001 \\
Globalidad negativa & $4.39(2.21)$ & $4.74(2.05)$ & .914 & .342 & .010 \\
\hline Na: RB
\end{tabular}

Nota: $\mathrm{RB}=$ Rendimiento Bajo; $\mathrm{RM}=$ Rendimiento Medio

\section{Discusión}

El primer objetivo pretendía explorar el poder predictivo de la motivación hacia el aprendizaje y las atribuciones evaluadas en el último curso de Educación Infantil sobre las habilidades matemáticas en $2^{\circ}$ de Primaria. En el presente estudio la motivación hacia el aprendizaje fue medida a través de las respuestas de los profesores a preguntas que incluían aspectos relacionados con creencias de autoeficacia, persistencia y actitud positiva, recogiendo así la multidimensionalidad de los aspectos que se incluyen en el interés para aprender (Wilson y Trainin, 2007). Los resultados expresan su peso significativo en la predicción de la totalidad de las habilidades matemáticas analizadas, con varianzas que oscilan, en conjunto, entre el $12.6 \%$ y el $28.2 \%$. Además, el peso de estas variables es ligeramente superior sobre las puntuaciones obtenidas en las habilidades matemáticas formales que implican el uso de símbolos matemáticos escritos (desarrolladas en contextos escolares), aspecto que puede estar relacionado con el buen hacer de los profesionales de esta etapa educativa.

La autocompetencia del niño es el predictor que mejor explica el rendimiento matemático en la gran mayoría de las variables analizadas. Asimismo, la actitud hacia el aprendizaje y la persistencia aportan también, aunque en menor medida, una contribución significativa adicional. La persistencia muestra un peso especialmente significativo en la predicción de los conocimientos conceptuales informales y las operaciones de cálculo con enunciado aritmético. Estos resultados van en la línea de investigaciones con niños más mayores (McKenzie et al., 2004; Moenikia y Zahed-Babelan, 2010; Pinxten et al., 2014; Suárez-Álvarez et al., 2014) y sugieren que el autoconcepto académico positivo y la orientación afectiva hacia el aprendizaje, generadas ya en la etapa de Educación Infantil a los 5 años, influyen sobre las metas y logros académicos futuros (Daniels, 2014; Fantuzzo et al., 2004; Ladd et al., 2000; McDermott, et al., 2001; Mokrova et al., 2013; Reimann et al., 2013) .

Continuando con el primer objetivo, al evaluar la capacidad predictiva de las dimensiones de estilo atribucional, solamente la dimensión internalidad positiva ha 
mostrado un peso significativo sobre el rendimiento académico, aunque con valores limitados. El presente estudio pone de manifiesto que en Educación Infantil las atribuciones parecen estar en una fase inicial, con una tendencia a asignar los éxitos académicos a factores internos mientras que los fracasos son todavía explicados de forma inconsistente en todas las dimensiones analizadas. Son necesarios más trabajos que profundicen en esta cuestión.

El conjunto de resultados obtenidos en relación al primer objetivo es coincidente con estudios realizados con muestras de mayor edad que sostienen que las variables motivacionales y atribucionales actúan como predictores sobre el rendimiento académico (Miñano y Castejón, 2008; Miñano y Castejón, 2011). No obstante, las varianzas obtenidas en estos estudios son muy superiores a las obtenidas en el presente trabajo. Probablemente, las diferencias entre ambos estudios son debidas, además de a la diversidad de los instrumentos utilizados, a la diferencia de edad entre ambas muestras, con grados de madurez, intereses y percepciones de autocompetencia muy dispares. Este hecho apuntaría a que el peso de la motivación sobre el rendimiento académico se incrementa con la edad. Una de las principales aportaciones del presente estudio es destacar que, aunque menor que en etapas posteriores, las variables motivacionales tiene un papel ya muy significativo en la etapa de Educación Infantil que debe ser tenido en cuenta.

El segundo objetivo pretendía estudiar en qué variables de motivación hacia el aprendizaje y de estilo atribucional evaluadas al finalizar la etapa de Educación Infantil diferían los alumnos con rendimiento matemático medio y con rendimiento matemático bajo en $2^{\circ}$ curso de Educación Primaria. En referencia las primeras, los alumnos con bajo rendimiento mostraron menores conductas de persistencia ante las dificultades que sus compañeros con rendimiento medio. La importancia de la persistencia en el adecuado desarrollo del aprendizaje escolar y sus dificultades se refleja también en el trabajo de Mokrova et al. (2013) con niños más pequeños. Mediante un diseño longitudinal estos investigadores encontraron que, independientemente de factores demográficos y de habilidades cognitivo-lingüísticas, los niños que eran más persistentes en completar una tarea desafiante a los 3 años mostraban mayores habilidades académicas (en lenguaje y matemáticas) dos años después.

Respecto a las dimensiones de estilo atribucional, los resultados muestran diferencias entre ambos grupos de rendimiento en el mismo sentido en la internalidad positiva. Es decir, los niños con rendimiento matemático bajo en $2^{\circ}$ curso de Primaria ya a los 5 años atribuían a sí mismos, en menor medida, los resultados positivos de sus acciones.

En síntesis, se puede concluir que los resultados obtenidos muestran que, ya en la etapa de Educación Infantil, las variables motivacionales hacia el aprendizaje, así como las atribuciones ante los resultados, influyen sobre el rendimiento futuro. Es más, se ha encontrado una clara relación entre puntuaciones bajas en algunas de estas variables, especialmente en la persistencia en las tareas, y los problemas de rendimiento matemático dos años después.

El presente estudio resulta pionero al demostrar de forma conjunta la importancia de las variables de motivación hacia el aprendizaje y de estilo atribucional tempranas para el rendimiento matemático posterior, aunque futuras investigaciones deberían incorporar otros procedimientos para la evaluación de la motivación a través del niño y las personas próximas a éste. Otra limitación es la ausencia en este estudio de otras variables explicativas del rendimiento matemático de índole personal (cognitivas, funcionamiento 
ejecutivo, etc.). Tampoco han sido tenidas en cuenta variables contextuales, tanto socioambientales (i.e. implicación de la familia en los resultados del aprendizaje escolar de sus hijos) como institucionales e instruccionales (i.e. interacciones dentro del contexto aula, expectativas del docente, etc.). Son necesarios estudios longitudinales para replicar y profundizar en los resultados alcanzados en este trabajo, teniendo en cuenta la contribución de las distintas variables de corte personal y contextual sobre el rendimiento. Igualmente son necesarias investigaciones dirigidas a precisar qué factores individuales y contextuales están en el origen de la motivación, especialmente en niños con pobres resultados académicos.

Entre las aplicaciones prácticas que se derivan del estudio, se apunta la necesidad de incorporar, ya desde la etapa de Educación Infantil, componentes de carácter motivacional para la formación del alumnado y del propio cuerpo docente. Es importante proporcionar a los educadores de Educación Infantil, y también a los padres, información sobre el papel de la motivación en el éxito escolar y sobre cómo desarrollar la motivación de sus alumnos/hijos, especialmente en niños con riesgo de dificultades. Como subraya un reciente meta-análisis el incremento de la motivación constituye uno de los factores que condiciona la eficacia de la intervención en estudiantes con dificultades matemáticas (Ise y Schulte-Körne, 2013).

Los maestros, y los padres, deben proporcionar ambientes de aprendizaje que promuevan la autonomía sobre el control externo. Cuando el aprendizaje se consigue con procedimientos que apoyan la implicación del niño se potencia el sentimiento de autodeterminación y la comprensión del material que debe aprender. Padres y educadores deben acompañar al niño en el proceso de aprendizaje transmitiendo su pasión y entusiasmo por conocer, potenciando sentimientos de autoeficacia y autocompetencia académica como base del éxito educativo.

\section{Referencias}

Bull, R., \& Lee, K. (2014). Executive functioning and mathematics achievement. Child Development Perspectives, 8(1), 36-41. doi: 10.1111/cdep.12059

Closas, A. H., Sanz de Acedo, M. L., y Ugarte, M. D. (2011). An explanatory model of the relations between cognitive and motivational variables and academic goals. Journal of Psychodidactics, 16(1), 19-38.

Conley, C. S., Haines, B. A., Hilt, L. M., \& Metalsky, G. I. (2001). The Children's Attributional Style Interview: Developmental tests of cognitive diathesis-stress theories of depression. Journal of Abnormal Child Psychology, 29(5), 445-463. doi: 10.1037/0022-3514.69.2.370

Daniels, D. H. (2014). Children's affective orientations in preschool and their initial adjustment to kindergarten. Psychology in the Schools, 51(3), 256-272. doi: $10.1002 /$ pits. 21748

Desoete, A., \& Grégoire, J. (2006). Numerical competence in young children and in children with mathematics learning disabilities. Learning and Individual Differences, 16(4), 351-367. doi: 10.1016/j.lindif.2006.12.006

Fantuzzo, J., Perry, M. A., \& McDermott, P. (2004). Preschool Approaches to Learning and Their Relationship to Other Relevant Classroom Competencies for LowIncome Children. School Psychology Quarterly, 19(3), 212. doi: 10.1521/scpq.19.3.212.40276 
Ginsburg, H. y Baroody, A. (2003). TEMA-3; Test de Competencia Matemática Básica. Madrid: TEA.

González, A. (2005). Motivación académica: Teoría, aplicación y evaluación. Madrid: Pirámide.

González-Pienda, J. A., Núñez, J. C., González-Pumariega, S., Roces, C., García, M., González, P., ... y Valle, A. (2000). Autoconcepto, proceso de atribución causal y metas académicas en niños con y sin dificultades de aprendizaje. Psicothema, 12(4), 548-556.

Ise, E., \& Schulte-Körne, G. (2013). Symptomatik, Diagnostik und Behandlung der Rechenstörung. Zeitschrift für Kinder- und Jugendpsychiatrie und Psychotherapie, 41(4), 271-282. doi: 10.1024/1422-4917/a000241

Ladd, G. W., Buhs, E. S., \& Seid, M. (2000). Children's initial sentiments about kindergarten: Is school liking an antecedent of early classroom participation and achievement?. Merrill-Palmer Quarterly (1982-), 46(2), 255-279.

Lozano, A. B., Pesutti, C. R., Blanco, J. C. B., y Canosa, S. S. (2000). Factores de atribución causal, enfoques de aprendizaje y rendimiento académico en el alumnado de educación secundaria de Galicia: datos para un análisis correlacional. Revista galego-portuguesa de psicoloxía e educación: revista de estudios e investigación en psicología y educación, 6, 792.

McDermott, P. A., Fantuzzo, J. W., Warley, H. P., Waterman, C., Angelo, L. E., Gadsden, V. L., \& Sekino, Y. (2011). Multidimensionality of teachers' graded responses for preschoolers' stylistic learning behavior: The Learning-To-Learn Scales. Educational and Psychological Measurement, 71(1), 148-169. doi: 10.1177/0013164410387351

McDermott, P. A., Goldberg, M. M., Watkins, M. W., Stanley, J. L., \& Glutting, J. J. (2006). A Nationwide Epidemiologic Modeling Study of LD Risk, Protection, and Unintended Impact. Journal of Learning Disabilities, 39(3), 230-251. doi: 10.1177/00222194060390030401

McDermott, P. A., Green, L. F., Francis, J. M., \& Stott, D. H. (2000). PLBS; Preschool Learning Behaviors Scale. Philadelphia: Edumetric \& Clinical Science.

McDermott, P. A., Mordell, M., \& Stoltzfus, J. C. (2001). The organization of student performance in American schools: Discipline, motivation, verbal learning, nonverbal learning. Journal of Educational Psychology, 93(1), 65-76. doi: 10.1037/0022-0663.93.1.65

McDermott, P. A., Rikoon, S. H., \& Fantuzzo, J. W. (2014). Tracing children's approaches to learning through Head Start, kindergarten, and first grade: Different pathways to different outcomes. Journal of Educational Psychology, 106(1), 200213. doi: $10.1037 / \mathrm{a} 0033547$

McKenzie, K., Gow, K., \& Schweitzer, R. (2004). Exploring first-year academic achievement through structural equation modelling. Higher Education Research \& Development, 23(1), 95-112. doi: 10.1016/j.lindif.2003.10.002

Miñano, P., y Castejón, J. L. (2008). Capacidad predictiva de las variables cognitivomotivacionales sobre el rendimiento académico. REME. Revista Electrónica de Motivación y Emoción, 28(11).

Miñano, P., y Castejón, J. L. (2011). Variables cognitivas y motivacionales en el rendimiento académico en Lengua y Matemáticas: un modelo estructural. Journal of Psychodidactics, 16(2), 203-230. doi: 10.1387/RevPsicodidact.930 
Miranda, A., García, R., Marco, R., \& Rosel, J. (2006). The role of the metacognitive beliefs system in learning disabilities in mathematics. Implications for intervention. In M. Veenman \& A. Desoete (Eds.), Metacognition and mathematics education (pp. 157-175). London: Nova Science Publisher.

Moenikia, M., \& Zahed-Babelan, A. (2010). A study of simple and multiple relations between mathematics attitude, academic motivation and intelligence quotient with mathematics achievement. Procedia-Social and Behavioral Sciences, 2(2), 15371542 .

Mokrova, I. L., O’Brien, M., Calkins, S. D., Leerkes, E. M., \& Marcovitch, S. (2013). The role of persistence at preschool age in academic skills at kindergarten. European journal of psychology of education, 28(4), 1495-1503.

Núñez, J. C., González-Pienda, J. A., González-Pumariega, S., Roces, C., Alvarez, L., González, P., ... y Rodríguez, S. (2005). Subgroups of attributional profiles in students with learning difficulties and their relation to self-concept and academic goals. Learning Disabilities Research \& Practice, 20(2), 86-97. doi: 10.1111/j.1540-5826.2005.00124.x

Op't Eynde, P., De Corte, E., \& Verschaffel, L. (2006). "Accepting emotional complexity": A socio-constructivist perspective on the role of emotions in the mathematics classroom. Educational Studies in Mathematics, 63(2), 193-207. doi: 10.1007/s10649-006-9034-4

Passolunghi, M. C., \& Lanfranchi, S. (2012). Domain-specific and domain-general precursors of mathematical achievement: A longitudinal study from kindergarten to first grade. British Journal of Educational Psychology, 82(1), 42-63. doi: 10.1111/j.2044-8279.2011.02039.x

Pasta, T., Mendola, M., Longobardi, C., Prino, L. E., \& Gastaldi, F. G. M. (2013). Attributional style of children with and without Specific Learning Disability. Electronic Journal of Research in Educational Psychology, 11(3), 649-664. doi: 10.1111/j.2044-8279.2011.02039.x

Pintrich, P. R., \& Schunk, D.H. (2006). Motivación en contextos educativos. Teoría, investigación y aplicaciones. Madrid: Pearson Education.

Pinxten, M., Marsh, H. W., De Fraine, B., Van Den Noortgate, W., \& Van Damme, J. (2014). Enjoying mathematics or feeling competent in mathematics? Reciprocal effects on mathematics achievement and perceived math effort expenditure. British Journal of Educational Psychology, 84(1), 152-174. doi: 10.1111/bjep.12028

Autores (2015). Competencias matemáticas y funcionamiento ejecutivo en preescolar: Evaluación clínica y ecológica. Revista de Psicodidáctica, 20(1), 65-85. doi: 10.1387/RevPsicodidact.11086

Reimann, G., Stoecklin, M., Lavallee, K., Gut, J., Frischknecht, M. C., \& Grob, A. (2013). Cognitive and motivational profile shape predicts mathematical skills over and above profile level. Psychology in the Schools, 50(1), 37-56. doi: 10.1002/pits.21659

Sarabia, A., y Iriarte, C. (2011). El aprendizaje de las matemáticas ¿Qué actitudes, creencias y emociones despierta esta materia en los alumnos? Navarra: Eunse.

Spreen, O., \& Strauss, E. (1991). A compendium of neuropsychological tests: administration, norms, and commentary. New York: Oxford University Press.

Suárez-Álvarez, J., Fernández-Alonso, R., \& Muñiz, J. (2014). Self-concept, motivation, expectations, and socioeconomic level as predictors of academic performance in 
mathematics. Learning and Individual Differences, 30, 118-123. doi: 10.1016/j.lindif.2013.10.019

Ugartetxea, J. (2002). La metacognición, el desarrollo de la autoeficacia y la motivación escolar. Revista de psicodidáctica, 13, 49-73.

Wechsler, D. (1996). WPPSI; Escala de Inteligencia de Wechsler para Preescolar y Primaria. Madrid: TEA Ediciones.

Weiner, B. (1986). An attributional theory of motivation and emotion. Springer Science \& Business Media.

Wigfield, A., \& Eccles, J. S. (2002). The development of competence beliefs, expectancies for success, and achievement values from childhood through adolescence. In A. Wigfield \& J. S. Eccles (Eds.), Development of achievement motivation (pp. 91-120).

Wilson, K., \& Trainin, G. (2007). First-grade students' motivation and achievement for reading, writing and spelling. Reading Psychology, 28(3), 257-282. doi: 10.1080/02702710601186464

Yen, C. J., Konold, T. R., \& McDermott, P. A. (2004). Does learning behavior augment cognitive ability as an indicator of academic achievement?. Journal of School Psychology, 42(2), 157-169. doi: 10.1016/j.jsp.2003.12.001

\section{Financiación}

Este trabajo está financiado por el Ministerio de Economía y Competitividad de España (EDU2012-37452) y la Universidad Jaume I (beca pre-doctoral 2I005PREDOC/2013/34).

\section{Autores}

Jessica Mercader Ruiz, licenciada en psicopedagogía, ha obtenido un máster en Neurociencia Cognitiva y Necesidades Educativas Específicas en la Universidad de Valencia. Actualmente, es becaria pre-doctoral en el programa de doctorado Intervención Social y Familiar de la Universidad Jaume I. Centra sus intereses en el área de Dificultades del Aprendizaje de las Matemáticas.

María-Jesús Presentación Herrero, doctora en psicología y profesora titular en el Departamento de Psicología Evolutiva, Educativa, Social y Metodología de la Universidad Jaume I de Castellón. Desde hace más de dos décadas su docencia y su investigación se han centrado en la intervención en las Dificultades del Aprendizaje y el TDAH.

Vicente Molinero Claramunt, maestro de Educación Primaria, ha obtenido un máster en Psicopedagogía en la Universidad Jaume I. Centra sus intereses en el área de Dificultades del Aprendizaje de las Matemáticas

Rebeca Siegenthaler Hierro, doctora en psicología y profesora ayudante doctora en el Departamento de Psicología Evolutiva, Educativa, Social y Metodología de la Universidad Jaume I. Es especialista en Dificultades de Aprendizaje y TDAH.

Ana Miranda Casas, es doctora en psicología y catedrática del Departamento de Psicología Evolutiva y de la Educación de la Universidad de Valencia. Tiene una extensa y fructífera trayectoria profesional, con múltiples investigaciones nacionales e internacionales sobre Dificultades del Aprendizaje y TDAH. 